\title{
AN ENHANCEMENT OF EDGE PRESERVATION IN OAMNHA DENOISING USING TEXTURE BOUNDARIES
}

Indupriya Kumarasamy Research Scholar, Department of Computer Science, Sri Ramakrishna College of Arts and Science, Coimbatore, Tamilnadu (India).

E-mail: indupriya1406@gmail.com

ORCID: https://orcid.org/0000-0003-0446-9490

Anna Saro Vijendran

Dean, School of Computing, Sri Ramakrishna College of

Arts and Science, Coimbatore, Tamilnadu (India).

E-mail: saroviji@rediffmail.com

ORCID: https://orcid.org/0000-0003-2200-1226

\section{Gitación sugerida:}

Kumarasamy, I., y Vijendran, A. S. (2021). An enhancement of edge preservation in OAMNHA denoising using texture boundaries. 3C Tecnología. Glosas de innovación aplicadas a la pyme, Edición Especial, (noviembre, 2021), 471-489. https://doi.org/10.17993/3ctecno.2021.specialissue8.471-489 


\section{ABSTRACT}

In image processing, the most challenging task is removing the noise from the images since the segmentation of image patches from noisy images has high complexity. To tackle this challenge, an Optimum Adaptive parameterized Mask Non-Harmonic Analysis (OAMNHA) based image denoising technique was proposed in Curvelet Transform (CT) domain. In this technique, the image was converted into frequency domain representation to decompose it into different subband. Then, edge-preserving segmentation using canny edge detection was applied for each subband to extract the edges and identify the edge regions from a noisy image. However, this process has high time consumption due to its complex computation. Hence in this article, a Neuro-Fuzzy (NF) methodology is proposed as edge-preserving segmentation method. Initially, noisy images represented in the frequency domain are given to the NF edge detector to segment the edge regions and homogeneous textures from a noisy image. Moreover, OAMNHA technique is applied for each region excluding edge regions to restore the noiseless image accurately. This segmentation of noisy images covers Neuro-fuzziness in the choice of the region boundary. Based on this segmentation, the boundary distortion is efficiently minimized since it defines texture boundaries with less time consumption and computational complexity. Also, the accuracy of edge-preserving segmentation is increased significantly. Finally, the experimental results prove that the proposed NF-OAMNHA-CT based image denoising technique has better performance than the OAMNHA-GT technique.

\section{KEYWORDS}

Image denoising, Edge-preserving segmentation, Canny edge detection, OAMNHA, Neuro-fuzzy approach. 


\section{INTRODUCTION}

Image denoising also known as noise reduction is the process of eradicating the noise from an image during edge preservation process. Normally, edge preserving process is one of the image processing techniques that remove noise or textures while preserving sharp edges. In these days, digital images play the most significant role in different applications such as magnetic satellite box, computer tomography, resonance imaging, and ecological systems. In general, the images are captured by the sensors. In some cases, like faulty components, data acquisition problems and normal phenomena interference, the most significant data can be degraded. As well, the noise may be occurred by the inaccurate compression and transmission. As a result, image denoising process is needed as primary process in the image processing to compensate the data corruption. Still, image denoising technique has lot of challenges since the artifacts are occurred during noise removal which blurs the images.

Hosotani et al. (2015) introduced an image denoising process with edge preserving and segmentation based on the Mask NHA (M- NHA). In this process, the zero-mean white Gaussian noise was eliminated by using the high-resolution frequency analysis. The regions including identical texture were analyzed on the noisy image. The non-uniform regions obtained by segmentation process were analyzed by using M-NHA to improve the Peak Signal-To-Noise Ratio (PSNR). Conversely, an optimization of the parameters used in the segmentation was not efficient and the threshold value for edge detection was fixed to suppress the unwanted data. Hence, an OAMNHA based image denoising technique was proposed by using SVM and firefly optimization algorithms. In this technique, Support Vector Machine (SVM) was applied for learning the parameters used in the segmentation and firefly algorithm was used for optimizing the threshold values for many noisy images. However, the efficiency of denoising was depending on the homogeneous texture segmentation since it uses feature vectors in spatial domain that cannot be provided to search similar patches from arbitrary regions. Therefore, the frequency domain coefficients have been used in image segmentation. Initially, the spatial domain image was transformed into frequency domains such as Wavelet Transform (OAMNHA-WT), Contourlet Transform (OAMNHA-CoT) and Curvelet Transform (OAMNHA-CT) separately. Then, the image was decomposed into different subband images and homogeneous patches were explored 
by using frequency coefficients for achieving segmentation process. At last, 2D NHA and inverse frequency transforms were applied independently for merging all subbands together.

Based on this technique, the noisy pixels are efficiently removed via segmentation process. Nonetheless, the region boundary was difficult to determine within a noisy image. The segmentation of noisy images has fuzziness in the collection of the region margin. It was assumed that fuzzy boundaries must be defined as edge regions. For this reason, canny edge detector was used for detecting the edge positions with homogeneous textures. But, the time consumption of this detection method was high due to its high computational complexity.

Therefore, in this article, an edge-preserving segmentation is performed based on the NF approach. In this technique, the considered noisy images denoted in the frequency domain transforms are applied to the NF edge detector to detect the edge regions and homogenous textures from a noisy image. This edge segmentation and detection has the Neuro-fuzziness in the choice of region boundary. Then, the edge regions are removed and the texture regions are used for restoring the noise-free images by applying the OAMNHA technique. Therefore, the boundary distortion is reduced by defining the texture boundaries due to segmentation and detection process. Also, the accuracy of the edge- preserving segmentation and detection is efficiently increased.

The remaining part of the article is organized as follows: Section II presents different image denoising approaches which are related to the proposed technique. Section III clarifies the methodology of proposed image denoising technique. Section IV illustrates the experimental results and Section $\mathrm{V}$ concludes the entire discussion.

Chen et al. (2013) proposed an edge preserving image denoising with a closed form solution. In this method, a novel pixel-based algorithm was proposed that formulates the image denoising problem as the Maximum-A-Posterior (MAP) estimation problem by using Markov Random Fields (MRF). This was converted into a continuous label assignment problem based on a Gaussian MRF model and then a closed form globally optimal solution was obtained. The pre- estimated image edge information was added into the energy function construction to preserve the image structures. Moreover, patch similarity based pairwise interaction was performed to better preserve the image details and achieve high robustness. However, Mean Squared Error (MSE) of this algorithm was high. 
Jiao and Huang (2014) proposed a new wavelet packet transform adaptive threshold image denoising method based on edge detection. By edge detection method, the wavelet packet coefficients corresponding to detected edge and other non-edge wavelet packet coefficients were treated by different threshold. By using the relativity among wavelet packet coefficients and neighbor dependency relation, the new variance neighbor estimate method was adopted and then the adaptive threshold was generated. On the other hand, the computational complexity of this method was high.

Gao, Wang and Liu (2015) proposed an image denoising method based on edge detection and pre-thresholding wiener filtering of multi-wavelets fusion for removing Gaussian noise from digital images. Initially, the noisy image was decomposed by using multiple wavelets and the edge of image was detected through wavelet multi-scale edge detection. Based on this, the wavelet coefficients belonging to the edge position were dealt with the improved wavelet threshold method whereas the others were dealt with the pre-thresholding wiener filtering. At last, the fusion algorithm was used based on wavelet analysis for obtaining the denoised image. However, the computational burden i.e., computation time was high and it requires an adaptive threshold selection using advanced optimization methods to further improve the image denoising performance.

Guo, Zhang and Zhang (2018) proposed an edge-preserved image denoising algorithm based on local adaptive regularization which can adaptively adjust denoising in accordance with different regions of noisy image by containing residual local energy function. In this method, detailed information of image was also well preserved at the process of denoising. However, this method has high time complexity. Yang et al. (2016) proposed a block thresholding image denoising using Dual-Tree Complex Wavelet Transform (DTCWT) for removing the additive white Gaussian noise from the images. Initially, DTCWT was applied on noisy image for obtaining the complex coefficients with properties of approximate shift invariance and directional selection. Moreover, block thresholding scheme was adopted in denoising process to select the optimal block size and threshold at each resolution level by reducing Stein's unbiased risk estimate. However, this method was suitable for preserving the uniform regions only. 
Jain and Tyagi (2017) proposed an adaptive edge-preserving image denoising technique by using patch-based weighted-Singular Value Decomposition (SVD) filtering in wavelet domain. In this filtering method, a group of non-local similar patches were considered for each local patch in the subband and the weights were estimated for this patch group based on the significance of its singular values. After that, these weights were used as a threshold for SVD filtering of the considered patch group. This process was applied to each local patch and all patches were aggregated together for obtaining the thresholder results in the subband. However, this algorithm was not able to obtain the depth of the image which has the shadow effect and the intensity values ranged in the black to gray.

Zhao and Shang (2018) proposed an adaptive edge-detection method i.e., a method of iterative threshold edge detection based on histogram. In this method, multi-scale wavelet transform was used for preprocessing the image in which the image detains were highlighted and the effect of manual setting filter coefficients was avoided. The variation of gray values between the pixels of local region was used for computing the gradients and the gradient directions were extended to four directions. The adaptive method was used for computing the threshold of edge-detection and the image was represented by histogram. After that, the ratio of the number of pixels in the bar and the total numbers of pixels were used for selecting the initial threshold.

The regions on both sides of the initial threshold were used for calculating the high threshold and low threshold iteratively. The detection errors, connection errors and the pseudo-edges caused by selecting the threshold artificially were avoided. However, the Signal-to-Noise Ratio (SNR) of this method was less. Guo et al. (2016) recommended a novel edge-preserving image denoising algorithm which treats the low and high-frequency components of the image separately. For restoration of high-frequency components, a neighborhood regression method was proposed. Moreover, an energy minimization function was developed for combining the low and high-frequency components composed for obtaining the result as denoised. However, the average run time of this algorithm was high and also the performance efficiency was not effective. 


\section{MATERIALS AND METHODS}

In this section, the proposed NF-OAMNHA-GT based image denoising technique using $\mathrm{NF}$ approach is explained briefly. Initially, the image $\mathrm{i}(\mathrm{x}, \mathrm{y})$ with size $\mathrm{M} \times \mathrm{N}$ is transformed into frequency domain such as CT. Then, the image is given to the NF edge detector to detect the edge regions and homogenous textures as follows:

The structure of the proposed Neuro Fuzzy edge detector shown in Figure 1(a) has four NF networks which are functioning as sub-detectors in the four directions, namely vertical, horizontal, right diagonal and left diagonal, respectively. Each sub-detector can operate on a window size of $3 \times 3$ which is shown in Figure 1(b). Also, each sub-detector estimates a different neighbourhood correlation between the center pixel of the filtering window and two of its neighbour's.

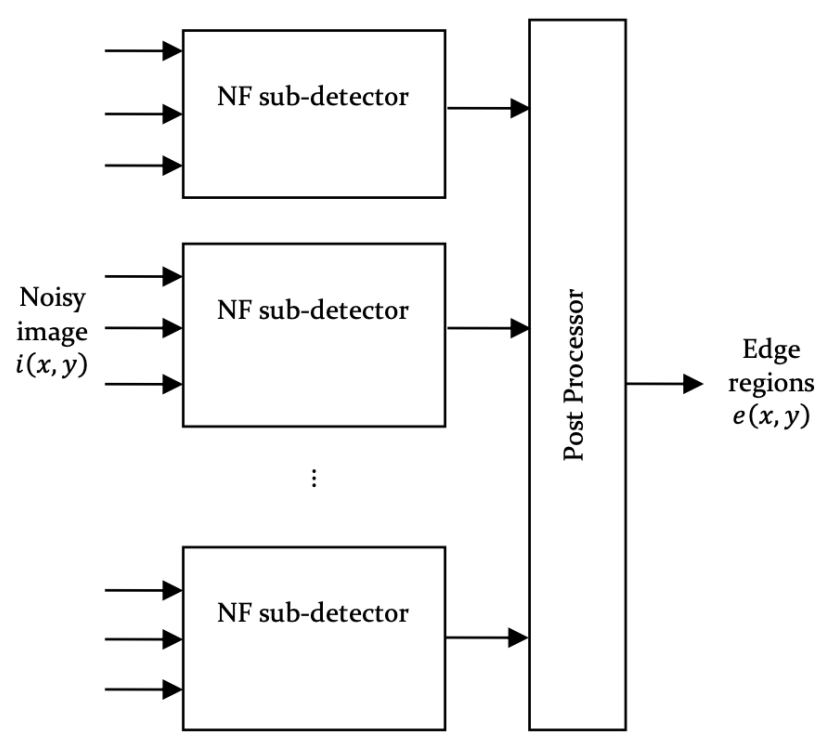

Figure 1(a). Structure of the NF Edge Detector.

Source: own elaboration.

Each NF sub-detector is a first-order Sugeno type Fuzzy Inference System (FIS) with 3-inputs and 1-output. Each input has 3 generalized bell type membership functions and the output has a linear membership function $(\mathrm{m})$. The input-output correlation of any of the NF sub-detectors is as follows: 
Consideri_1,i_2,i_3 are the inputs of the NF sub-detector and e is its output. Each possible combination of inputs and their related membership functions is denoted by a rule in the rule base of the NF sub-detector. As the NF sub-detector consists of 3 inputs and each input has 3 membership functions, the rule base has 27 rules totally which are given below:

$$
\begin{aligned}
& \text { if }\left(i_{1} \text { is } m_{11}\right) \text { and }\left(i_{2} \text { is } m_{21}\right) \text { and }\left(i_{3} \text { is } m_{31}\right) \text {, then } \mathrm{R}_{1}=\mathrm{F}_{1}\left(\mathrm{i}_{1}, \mathrm{i}_{2}, \mathrm{i}_{3}\right) \\
& \text { if }\left(\mathrm{i}_{1} \text { is } \mathrm{m}_{11}\right) \text { and }\left(\mathrm{i}_{2} \text { is } \mathrm{m}_{21}\right) \text { and }\left(\mathrm{i}_{3} \text { is } \mathrm{m}_{32}\right) \text {, then } \mathrm{R}_{2}=\mathrm{F}_{2}\left(\mathrm{i}_{1}, \mathrm{i}_{2}, \mathrm{i}_{3}\right) \\
& \text { if }\left(\mathrm{i}_{1} \text { is } \mathrm{m}_{11}\right) \text { and }\left(\mathrm{i}_{2} \text { is } \mathrm{m}_{21}\right) \text { and }\left(\mathrm{i}_{3} \text { is } \mathrm{m}_{33}\right) \text {, then } \mathrm{R}_{3}=\mathrm{F}_{3}\left(\mathrm{i}_{1}, \mathrm{i}_{2}, \mathrm{i}_{3}\right) \\
& \text { if }\left(\mathrm{i}_{1} \text { is } \mathrm{m}_{11}\right) \text { and }\left(\mathrm{i}_{2} \text { is } \mathrm{m}_{22}\right) \text { and }\left(\mathrm{i}_{3} \text { is } \mathrm{m}_{31}\right) \text {, then } \mathrm{R}_{4}=\mathrm{F}_{4}\left(\mathrm{i}_{1}, \mathrm{i}_{2}, \mathrm{i}_{3}\right)
\end{aligned}
$$

if $\left(i_{1}\right.$ is $\left.m_{13}\right)$ and $\left(i_{2}\right.$ is $\left.m_{23}\right)$ and $\left(i_{3}\right.$ is $\left.m_{33}\right)$, then $R_{27}=F_{27}\left(i_{1}, i_{2}, i_{3}\right)$

Here, $m_{i j}$ is the $j^{\text {th }}$ membership function of the $i^{\text {th }}$ input, $R_{k}$ is the output of the $k^{\text {th }}$ rule and $\mathrm{F}_{\mathrm{k}}$ is the $\mathrm{k}^{\text {th }}$ output membership function. The input membership functions $\left(\mathrm{m}_{\mathrm{ij}}\right)$ are generalized bell type which is defined as follows:

$$
m_{i j}=\frac{1}{1+\left|\frac{i_{i}-a_{i j}}{b_{i j}}\right|^{2 c_{i j}}}
$$

The output membership functions $(\mathrm{Fk})$ are as:

$\mathrm{F}_{\mathrm{k}}=\mathrm{d}_{\mathrm{k} 0}+\mathrm{d}_{\mathrm{k} 1} \mathrm{i}_{1}+\mathrm{d}_{\mathrm{k} 2} \mathrm{i}_{2}+\mathrm{d}_{\mathrm{k} 3} \mathrm{i}_{3}$

For $\mathrm{i}=\mathrm{j}=1,2,3$ and $\mathrm{k}=1,2, \ldots, 27$

The factors a, b, c and $\mathrm{d}$ in the above equations are constants that differentiate the shape of the membership functions. The optimal values of these parameters are determined by learning process. Each NF sub-detector is learned individually. The setup used for learning is shown in Figure 2.

Here, the parameters of the Neuro Fuzzy sub-detectors under learning are iteratively fine-tuned so that its output converges to the output of the ideal edge detector which can 
properly detect the positions of the edge pixels in the given image. The ideal edge detector output represented by the target training image is used only for learning process.

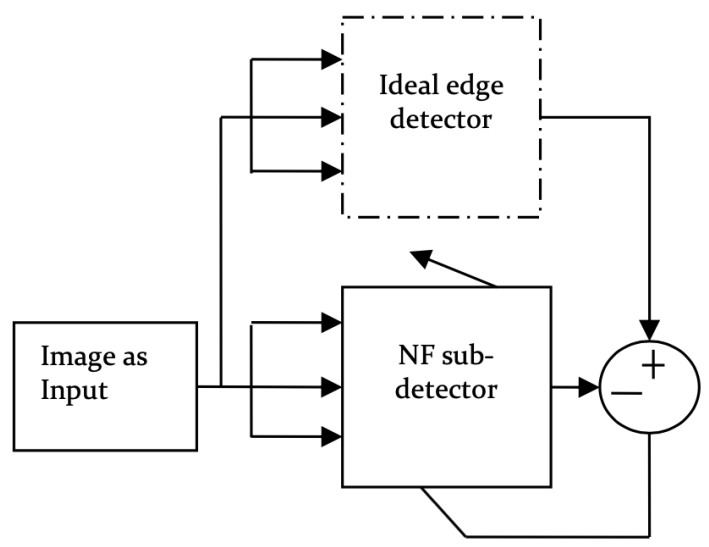

Figure 2. Training of the Sub-detectors.

Source: own elaboration.

The output of the Neuro Fuzzy sub-detector is the weighted average of the individual rule outputs. The weighting factor $\left(\mathrm{w}_{\mathrm{k}}\right)$ of each rule is computed by estimating the membership expressions in the antecedent of the rule. This is achieved by converting the input values to fuzzy membership values by using the input membership functions and applying and operator to these membership values. Thus, the weighting factors of the rules are computed as follows:

$$
\begin{gathered}
\mathrm{w}_{1}=\mathrm{m}_{11}\left(\mathrm{i}_{1}\right) \cdot \mathrm{m}_{21}\left(\mathrm{i}_{2}\right) \cdot \mathrm{m}_{31}\left(\mathrm{i}_{3}\right) \\
\mathrm{w}_{2}=\mathrm{m}_{11}\left(\mathrm{i}_{1}\right) \cdot \mathrm{m}_{21}\left(\mathrm{i}_{2}\right) \cdot \mathrm{m}_{32}\left(\mathrm{i}_{3}\right) \\
\mathrm{w}_{3}=\mathrm{m}_{11}\left(\mathrm{i}_{1}\right) \cdot \mathrm{m}_{21}\left(\mathrm{i}_{2}\right) \cdot \mathrm{m}_{33}\left(\mathrm{i}_{3}\right) \\
\cdot \\
\cdot \\
\mathrm{w}_{27}=\mathrm{m}_{13}\left(\mathrm{i}_{1}\right) \cdot \mathrm{m}_{23}\left(\mathrm{i}_{2}\right) \cdot \mathrm{m}_{33}\left(\mathrm{i}_{3}\right)
\end{gathered}
$$

After computing the weighting factors, the output of the NF sub-detector can be obtained by computing the weighted average of the individual rule outputs as: 


$$
\mathrm{e}=\frac{\sum_{\mathrm{k}=1}^{27} \mathrm{w}_{\mathrm{k}} \mathrm{R}_{\mathrm{k}}}{\sum_{\mathrm{k}=1}^{27} \mathrm{w}_{\mathrm{k}}}
$$

The outputs of the Neuro Fuzzy sub-detectors values are given to the postprocessor to generate the final NF network output. Normally, the postprocessor is used for computing the average value of the sub-detector outputs and comparing this value with a threshold. The threshold value is half of the available dynamic range for the pixel luminance values. For 8-bit images where the pixel values range between 0 and 255, the threshold value is taken as 128. The input and output correlation of the postprocessor is described as follows:

Consider $\mathrm{e}_{1}, \mathrm{e}_{2}, \ldots, \mathrm{e}_{1}$ is the outputs of the $\mathrm{NF}$ sub-detectors where $\mathrm{l}$ is the number of $\mathrm{NF}$ sub-detectors used. The output of the postprocessor is computed in two steps:

Step 1: The average value of the individual NF sub-detectors outputs is computed as:

$$
\mathrm{e}_{\mathrm{AV}}=\frac{1}{\mathrm{l}} \sum_{\mathrm{k}=1}^{\mathrm{l}} \mathrm{e}_{\mathrm{k}}
$$

Step 2: The computed $\mathrm{e}_{\mathrm{AV}}$ value is altered into 0 (black) or 255 (white) by relating it with the threshold as:

$$
\mathrm{e}(\mathrm{x}, \mathrm{y})=\left\{\begin{array}{cc}
0, & \text { if } \mathrm{e}_{\mathrm{AV}}<128 \\
255, & \text { Otherwise }
\end{array}\right.
$$

In equation (6), $\mathrm{e}(\mathrm{x}, \mathrm{y})$ represents the result of the postprocessor i.e., the output of the Neuro Fuzzy edge detector that denotes the conclusion whether the center pixel of the filtering window is an edge pixel or not. This is continued until all pixels of the noisy image are classified properly. Thus, the edges from the noisy image are extracted efficiently and then the edge regions are defined accurately. After that, the texture boundaries formed and OAMNHA technique is applied for each homogeneous texture region in order to reconstruct the noise-free images with the increased segmentation accuracy and reduced computational complexity. 


\section{RESULTS}

In this section, the performance of proposed NF-OAMNHA-CT based image denoising technique is evaluated and compared with the existing OAMNHA-CT technique using MATLAB. In this experiment, target images with various features are gathered from LIVE (The Laboratory for Image Video Engineering) image database at the University of Texas at Austin. The comparison is obtainable in terms of PSNR, Mean Absolute Error (MAE) and Structural Similarity Index (SSIM). The results of proposed NF-OAMNHA-CT based image denoising technique are shown in Figure 3.

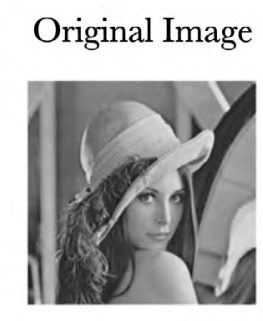

Denoised Image using OAMNHA-CT

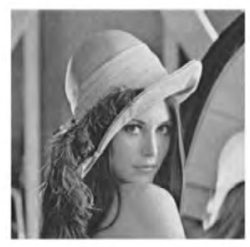

Noisy Image

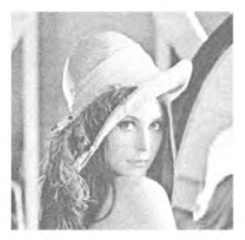

Denoised Image using NF-

OAMNHA-CT

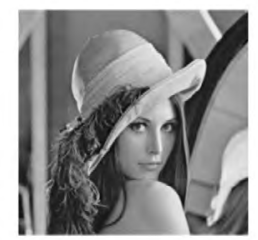

Figure 3. Results of Proposed and Existing Denoising Technique for Lena Image.

Source: own elaboration.

\subsection{PSNR}

It is the ratio of the maximum signal power to noise power and computed as:

PSNR $=10 \log _{10} \frac{255^{2}}{\mathrm{MSE}}$

Where, MSE $=\frac{1}{\mathrm{~m} n} \sum_{\mathrm{i}=1}^{\mathrm{m}-1} \sum_{\mathrm{j}=1}^{\mathrm{n}-1}\left(\mathrm{x}_{\mathrm{ij}}-\mathrm{y}_{\mathrm{ij}}\right)^{2}$

The comparison of denoising PSNR which is made between proposed NF-OAMNHA-CT and existing OAMNHA-GT techniques is shown in Table 1. It shows that the proposed NF-OAMNHA-GT technique achieves higher PSNR compared with the OAMNHA-CT 
technique for image denoising. For example, consider the statue with $\sigma=5$. Then, the PSNR of proposed NF-OAMNHA-CT technique is $4.5 \%$ higher than OAMNHA-CT technique. Table 1. Comparison of denoising PSNR results ( $\sigma$ : Noise density).

\begin{tabular}{|c|c|c|c|c|}
\hline \multirow{2}{*}{$\sigma$} & \multicolumn{2}{|c|}{ Cameraman } & \multicolumn{2}{|c|}{ Bikes } \\
\hline & OAMNHA-CT & NF-OAMNHA-CT & OAMNHA-CT & NF-OAMNHA-CT \\
\hline 5 & 43.88 & 46.25 & 44.86 & 46.62 \\
\hline 10 & 42.33 & 45.21 & 40.68 & 42.86 \\
\hline 15 & 39.14 & 41.38 & 38.99 & 41.03 \\
\hline 20 & 38.86 & 40.48 & 36.07 & 38.55 \\
\hline 25 & 36.72 & 39.06 & 35.66 & 37.39 \\
\hline 30 & 35.69 & 38.13 & 34.53 & 36.72 \\
\hline \multirow{2}{*}{$\sigma$} & \multicolumn{2}{|c|}{ Ocean } & \multicolumn{2}{|c|}{ Statue } \\
\hline & OAMNHA-CT & NF-OAMNHA-CT & OAMNHA-CT & NF-OAMNHA-CT \\
\hline 5 & 45.99 & 48.03 & 45.98 & 48.05 \\
\hline 10 & 41.67 & 43.49 & 41.86 & 43.61 \\
\hline 15 & 40.06 & 42.99 & 40.04 & 42.89 \\
\hline 20 & 38.35 & 40.11 & 39.22 & 41.33 \\
\hline 25 & 36.88 & 38.62 & 36.93 & 38.74 \\
\hline 30 & 35.96 & 37.85 & 35.76 & 37.92 \\
\hline \multirow{2}{*}{$\sigma$} & \multicolumn{2}{|c|}{ Barbara } & \multicolumn{2}{|c|}{ Lena } \\
\hline & OAMNHA-CT & NF-OAMNHA-CT & OAMNHA-CT & NF-OAMNHA-CT \\
\hline 5 & 45.91 & 47.68 & 46.32 & 48.86 \\
\hline 10 & 42.03 & 44.21 & 42.98 & 44.37 \\
\hline 15 & 39.81 & 41.19 & 41.05 & 43.22 \\
\hline 20 & 38.94 & 40.06 & 39.86 & 42.31 \\
\hline 25 & 36.97 & 38.34 & 39.17 & 41.95 \\
\hline 30 & 35.59 & 37.95 & 37.99 & 39.04 \\
\hline \multirow{2}{*}{$\sigma$} & \multicolumn{2}{|c|}{ Coinsfountain } & \multicolumn{2}{|c|}{ Lighthouse } \\
\hline & OAMNHA-CT & NF-OAMNHA-CT & OAMNHA-CT & NF-OAMNHA-CT \\
\hline 5 & 44.92 & 46.28 & 45.90 & 47.68 \\
\hline 10 & 41.77 & 43.51 & 40.68 & 42.31 \\
\hline 15 & 38.25 & 40.85 & 39.17 & 41.85 \\
\hline 20 & 36.88 & 38.43 & 36.89 & 38.47 \\
\hline 25 & 35.06 & 38.36 & 35.95 & 37.52 \\
\hline 30 & 34.18 & 36.74 & 35.06 & 37.19 \\
\hline \multirow{2}{*}{$\boldsymbol{\sigma}$} & \multicolumn{4}{|c|}{ Stream } \\
\hline & \multicolumn{2}{|c|}{ OAMNHA-CT } & \multicolumn{2}{|c|}{ NF-OAMNHA-CT } \\
\hline 5 & \multicolumn{2}{|c|}{40.97} & \multicolumn{2}{|c|}{43.06} \\
\hline 10 & \multicolumn{2}{|c|}{37.81} & \multicolumn{2}{|c|}{39.65} \\
\hline 15 & \multicolumn{2}{|c|}{36.07} & \multicolumn{2}{|c|}{38.71} \\
\hline
\end{tabular}




\begin{tabular}{|l|l|l|}
\hline 20 & 34.15 & 36.34 \\
\hline 25 & 41.92 & 43.87 \\
\hline 30 & 41.28 & 43.43 \\
\hline
\end{tabular}

Source: own elaboration.

\subsection{MAE}

It is the measure used to calculating the average magnitude of error in the prediction groups and computed as:

$$
\operatorname{MAE}=\frac{1}{\mathrm{n}} \sum_{\mathrm{i}=1}^{\mathrm{n}}\left|\mathrm{P}_{\mathrm{i}}-\mathrm{Y}_{\mathrm{i}}\right|=\frac{1}{\mathrm{n}} \sum_{\mathrm{i}=1}^{\mathrm{n}}\left|\varepsilon_{\mathrm{i}}\right|
$$

In equation (9), $\mathrm{P}_{\mathrm{i}}$ is the prediction value, $\mathrm{Y}_{\mathrm{i}}$ is the true value and $\varepsilon_{\mathrm{i}}$ is the absolute error. The comparison of denoising MAE for proposed NF-OAMNHA-CT and the existing OAMNHA-CT techniques is shown in Table 2. It shows that the proposed NF-OAMNHAGT technique has less MAE than the OAMNHA-CT technique for image denoising. If the statue with $\sigma=5$, then the MAE of proposed NF-OAMNHA-CT technique is $26.67 \%$ less than OAMNHA-CT technique.

Table 2. Comparison of denoising MAE results.

\begin{tabular}{|c|c|c|c|c|}
\hline \multirow{2}{*}{$\sigma$} & \multicolumn{2}{|c|}{ Cameraman } & \multicolumn{2}{|c|}{ Bikes } \\
\hline & OAMNHA-CT & NF-OAMNHA-CT & OAMNHA-CT & NF-OAMNHA-CT \\
\hline 5 & 1.65 & 1.12 & 1.70 & 1.24 \\
\hline 10 & 2.55 & 1.96 & 3.65 & 3.18 \\
\hline 15 & 3.44 & 2.91 & 4.81 & 4.36 \\
\hline 20 & 4.29 & 3.73 & 6.06 & 5.49 \\
\hline 25 & 5.51 & 5.05 & 7.46 & 6.93 \\
\hline 30 & 5.68 & 5.17 & 8.58 & 8.01 \\
\hline \multirow{2}{*}{$\sigma$} & \multicolumn{2}{|c|}{ Ocean } & \multicolumn{2}{|c|}{ Statue } \\
\hline & OAMNHA-CT & NF-OAMNHA-CT & OAMNHA-CT & NF-OAMNHA-CT \\
\hline 5 & 1.83 & 1.35 & 1.95 & 1.43 \\
\hline 10 & 2.99 & 2.47 & 2.96 & 2.46 \\
\hline 15 & 3.92 & 3.39 & 3.87 & 3.33 \\
\hline 20 & 4.28 & 3.71 & 4.52 & 4.09 \\
\hline 25 & 5.08 & 4.56 & 5.20 & 4.75 \\
\hline 30 & 5.63 & 5.12 & 5.74 & 5.27 \\
\hline \multirow{2}{*}{$\sigma$} & \multicolumn{2}{|c|}{ Barbara } & \multicolumn{2}{|c|}{ Lena } \\
\hline & OAMNHA-CT & NF-OAMNHA-CT & OAMNHA-CT & NF-OAMNHA-CT \\
\hline 5 & 2.13 & 1.68 & 1.99 & 1.53 \\
\hline 10 & 3.25 & 2.71 & 2.81 & 2.38 \\
\hline
\end{tabular}




\begin{tabular}{|c|c|c|c|c|}
\hline 15 & 3.96 & 3.43 & 3.44 & 2.91 \\
\hline 20 & 4.68 & 4.16 & 3.88 & 3.54 \\
\hline 25 & 5.26 & 4.72 & 4.36 & 3.87 \\
\hline 30 & 5.90 & 5.45 & 4.78 & 4.29 \\
\hline \multirow{2}{*}{$\boldsymbol{\sigma}$} & \multicolumn{2}{|c|}{ Coinsfountain } & \multicolumn{2}{|c|}{ Lighthouse } \\
\hline & OAMNHA-CT & NF-OAMNHA-CT & OAMNHA-CT & NF-OAMNHA-CT \\
\hline 5 & 2.54 & 2.08 & 2.25 & 1.73 \\
\hline 10 & 3.96 & 3.42 & 3.31 & 2.86 \\
\hline 15 & 5.18 & 4.67 & 4.28 & 3.74 \\
\hline 20 & 6.24 & 5.71 & 4.98 & 4.51 \\
\hline 25 & 7.07 & 6.64 & 5.60 & 5.49 \\
\hline 30 & 7.89 & 7.53 & 6.05 & 5.52 \\
\hline \multirow{2}{*}{$\sigma$} & \multicolumn{4}{|c|}{ Stream } \\
\hline & \multicolumn{2}{|c|}{ OAMNHA-CT } & \multicolumn{2}{|c|}{ NF-OAMNHA-CT } \\
\hline 5 & \multicolumn{2}{|c|}{3.27} & \multicolumn{2}{|c|}{2.76} \\
\hline 10 & \multicolumn{2}{|c|}{5.52} & \multicolumn{2}{|c|}{5.04} \\
\hline 15 & \multicolumn{2}{|c|}{7.34} & \multicolumn{2}{|c|}{6.88} \\
\hline 20 & \multicolumn{2}{|c|}{8.74} & \multicolumn{2}{|c|}{8.25} \\
\hline 25 & \multicolumn{2}{|c|}{9.98} & \multicolumn{2}{|c|}{9.41} \\
\hline 30 & \multicolumn{2}{|c|}{10.90} & \multicolumn{2}{|c|}{10.53} \\
\hline
\end{tabular}

Source: own elaboration.

\subsection{SSIM}

It is the similarity measure between any two images $\mathrm{i}(\mathrm{x}, \mathrm{y})$ and computed as follows:

$$
\operatorname{SSIM}(x, y)=\frac{\left(2 \mu_{x} \mu_{y}+c_{1}\right)\left(2 \sigma_{x y}+c_{2}\right)}{\left(\mu_{x}^{2}+\mu_{y}^{2}+c_{1}\right)\left(\sigma_{x}^{2}+\sigma_{y}^{2}+c_{2}\right)}
$$

In equation (10), the averages of $x$ and $y$ are represented as $\mu_{x}$ and $\mu_{y}$. Also, the variances of $\mathrm{x}$ and $\mathrm{y}$ are represented as $\sigma_{\mathrm{x}}{ }^{2}$ and $\sigma_{\mathrm{y}}{ }^{2} \mathrm{x}, \mathrm{y}$ respectively, $\mathrm{c}_{1}$ and $\mathrm{c}_{2}$ are constants. As well, the covariance of $\mathrm{x}$ and $\mathrm{y}$ is denoted as $\sigma_{\mathrm{xy}}$.

The comparison of denoising SSIM for both proposed NF-OAMNHA-CT and the existing OAMNHA-CT technique is shown in Table 3. It shows that the proposed NF-OAMNHACT technique has higher SSIM compared to the OAMNHA-CT based image denoising technique. For example, consider the statue with $\sigma=5$. Then, the SSIM of proposed NFOAMNHA-CT technique is $0.31 \%$ higher than OAMNHA-CT technique.

Table 3. Comparison of denoising SSIM results. 


\begin{tabular}{|c|c|c|c|c|}
\hline \multirow{2}{*}{$\sigma$} & \multicolumn{2}{|c|}{ Cameraman } & \multicolumn{2}{|c|}{ Bikes } \\
\hline & OAMNHA-CT & NF-OAMNHA-CT & OAMNHA-CT & NF-OAMNHA-CT \\
\hline 5 & 0.984 & 0.987 & 0.992 & 0.995 \\
\hline 10 & 0.966 & 0.969 & 0.980 & 0.983 \\
\hline 15 & 0.942 & 0.945 & 0.949 & 0.952 \\
\hline 20 & 0.896 & 0.899 & 0.914 & 0.917 \\
\hline 25 & 0.876 & 0.879 & 0.866 & 0.869 \\
\hline 30 & 0.865 & 0.868 & 0.825 & 0.828 \\
\hline \multirow{2}{*}{$\sigma$} & \multicolumn{2}{|c|}{ Ocean } & \multicolumn{2}{|c|}{ Statue } \\
\hline & OAMNHA-CT & NF-OAMNHA-CT & OAMNHA-CT & NF-OAMNHA-CT \\
\hline 5 & 0.985 & 0.988 & 0.978 & 0.981 \\
\hline 10 & 0.958 & 0.961 & 0.954 & 0.957 \\
\hline 15 & 0.901 & 0.904 & 0.917 & 0.920 \\
\hline 20 & 0.866 & 0.869 & 0.888 & 0.891 \\
\hline 25 & 0.827 & 0.830 & 0.865 & 0.868 \\
\hline 30 & 0.806 & 0.809 & 0.846 & 0.849 \\
\hline \multirow{2}{*}{$\sigma$} & \multicolumn{2}{|c|}{ Barbara } & \multicolumn{2}{|c|}{ Lena } \\
\hline & OAMNHA-CT & NF-OAMNHA-CT & OAMNHA-CT & NF-OAMNHA-CT \\
\hline 5 & 0.985 & 0.988 & 0.968 & 0.971 \\
\hline 10 & 0.966 & 0.969 & 0.943 & 0.946 \\
\hline 15 & 0.953 & 0.956 & 0.927 & 0.930 \\
\hline 20 & 0.939 & 0.942 & 0.910 & 0.913 \\
\hline 25 & 0.917 & 0.920 & 0.896 & 0.899 \\
\hline 30 & 0.911 & 0.914 & 0.884 & 0.887 \\
\hline \multirow{2}{*}{$\sigma$} & \multicolumn{2}{|c|}{ Coinsfountain } & \multicolumn{2}{|c|}{ Lighthouse } \\
\hline & OAMNHA-CT & NF-OAMNHA-CT & OAMNHA-CT & NF-OAMNHA-CT \\
\hline 5 & 0.986 & 0.989 & 0.976 & 0.979 \\
\hline 10 & 0.956 & 0.959 & 0.947 & 0.950 \\
\hline 15 & 0.921 & 0.924 & 0.927 & 0.930 \\
\hline 20 & 0.874 & 0.877 & 0.880 & 0.883 \\
\hline 25 & 0.826 & 0.829 & 0.867 & 0.870 \\
\hline 30 & 0.794 & 0.797 & 0.838 & 0.841 \\
\hline \multirow{2}{*}{$\sigma$} & \multicolumn{4}{|c|}{ Stream } \\
\hline & \multicolumn{2}{|c|}{ OAMNHA-CT } & \multicolumn{2}{|c|}{ NF-OAMNHA-CT } \\
\hline 5 & \multicolumn{2}{|c|}{0.993} & \multicolumn{2}{|c|}{0.996} \\
\hline 10 & \multicolumn{2}{|c|}{0.972} & \multicolumn{2}{|c|}{0.975} \\
\hline 15 & \multicolumn{2}{|c|}{0.913} & \multicolumn{2}{|c|}{0.916} \\
\hline 20 & \multicolumn{2}{|c|}{0.842} & \multicolumn{2}{|c|}{0.845} \\
\hline 25 & \multicolumn{2}{|c|}{0.778} & \multicolumn{2}{|c|}{0.781} \\
\hline 30 & \multicolumn{2}{|c|}{0.722} & & \\
\hline
\end{tabular}

Source: own elaboration. 


\section{CONCLUSIONS}

In this paper, NF-based edge-preserving segmentation is proposed to enhance the OAMNHA-based image denoising technique in different frequency transform domains. Initially, the considered image is fed into the Neuro Fuzzy edge detector to classify the pixel of the given image is whether edge pixel or not. For all pixels in the image, Neuro Fuzzy filtering process is repetitive and the edges are extracted precisely from the noisy image. Then, the extracted edges are defined as the edge regions. Also, the texture boundaries are constructed based on the homogeneous texture segmentation. For each segment, OAMNHA technique is applied to restore the noiseless image efficiently. Therefore, the time consumption for edge detection is reduced and the accuracy of the segmentation process is also increased. Finally, the experimental results proved that the proposed NF-OAMNHACT technique achieves higher PSNR, SSIM and less MAE than the OAMNHA-CT based image denoising technique. In many applications, e.g., medical or satellite imaging, the edges are key features and thus must be preserved sharp and undistorted in smoothing/ denoising. Also, edge preserving denoising is useful for the images capturing from camera which is an optical device and prone to sensor noise, especially in dark environments or environments with extreme high dynamic range.

\section{ACKNOWLEDGEMENT}

With pride and pleasure, I use this opportunity to place on record a deep sense of gratitude to my guiding light Dr. ANNA SARO VIJENDRAN, M.C.A., M. Phil., Ph. D., Dean, School of Computing, Sri Ramakrishna College of Arts \& Science (Autonomous), for her guidance and help throughout this research work and in writing this research paper. I deeply express my cordial thanks to all the Faculty Members of Department of Computer Science and Librarians of Sri Ramakrishna College of Arts \& Science (Autonomous).

\section{REFERENCES}

Chen, S., Liu, M., Zhang, W., \& Liu, J. (2013). Edge preserving image denoising with a closed form solution. Pattern Recognition, 46(3), 976-988. https://doi.org/10.1016/j. patcog.2012.08.014 
Gao, L., Wang, G., \& Liu, J. (2015). Image denoising based on edge detection and prethresholding Wiener filtering of multi-wavelets fusion. International fournal of Wavelets, Multiresolution and Information Processing, 13(05), 1550031. https://doi. org/10.1142/S0219691315500319

Guo, F., Zhang, G., \& Zhang, M. (2018). Edge-preserving image denoising. IET Image Processing, 12(8), 1394-1401. https://doi.org/10.1049/iet-ipr.2017.0880

Guo, L., Chen, W., Liao, Y., Liao, H., \& Li, J. (2016). An edge preserved image denoising algorithm based on local adaptive regularization. Fournal of Sensors, 2016. Article ID 2019569. http://dx.doi.org/10.1155/2016/2019569

Hosotani, F., Inuzuka, Y., Hasegawa, M., Hirobayashi, S., \& Misawa, T. (2015). Image Denoising With Edge-Preserving and Segmentation Based on Mask NHA. IEEE Transactions on Image Processing, 24(12), 6025-6033. https://doi.org/10.1109/ TIP.2015.2494461

Indupriya, K., \& Kumar, P. R. (2017). An Optimum Adaptive Parameterized Mask NHA Based Image Denoising. International Fournal of Engineering and Technology (IfET), 9(3), 2389-2399. https://doi.org/10.21817/ijet/2017/v9i3/1709030190

Jain, P., \& Tyagi, V. (2017). An adaptive edge-preserving image denoising technique using patch-based weighted-SVD filtering in wavelet domain. Multimedia Tools and Applications, 76(2), 1659-1679. https://doi.org/10.1007/s11042-015-3154-8

Jiao, Y., \& Huang, B. W. (2014). A new adaptive threshold image denoising method based on edge detection. Applied Mechanics and Materials, 678, 137-142. https://doi. org/10.4028/www.scientific.net/AMM.678.137

Vijendran, A. S., \& Kumarasamy, I. (2018). Image Denoising by Optimum Adaptive Parameterized Mask NHA in Various Frequency Domains. International fournal of Pure and Applied Mathematics, 119(18), 3405-3413. https://www.researchgate. net/publication/327765365_Image_Denoising_by_Optimum_Adaptive_ Parameterized_Mask_NHA_in_Various_Frequency_Domains 
Yang, P., Yang, G., Gai, S., \& Chen, J. (2016). Block thresholding image denoising with dual-tree complex wavelet transform. Fournal of Intelligent $\&$ Fuzzy Systems, 30(6), 3197-3207. https://doi.org/10.3233/IFS-152063

Zhao, H., \& Shang, M. (2018). An adaptive edge-detection method based on histogram. Modern Physics Letters B, 32(34n36), 1840088. https://doi.org/10.1142/ S0217984918400882 
